\title{
Oncologic Outcomes of Breast Cancer Patients Treated with Oncoplastic Surgery
}

\author{
Leda Marques Ribeiro ${ }^{1}$, Daniela Francescato Veiga ${ }^{2,3 *}$, Ivanildo Archangelo-Junior ${ }^{1}$, \\ Fabíola Soares Moreira Campos ${ }^{1}$, Renata Bites Amorim ${ }^{1}$, Mírian Fátima Brasil Engelman ${ }^{4}$, \\ Yara Juliano ${ }^{5}$, Lydia Masako Ferreira ${ }^{3}$
}

${ }^{1}$ Division of Mastology, Department of Gynecology, Universidade do Vale do Sapucaí, Pouso Alegre, Brazil; ${ }^{2}$ Division of Plastic Surgery, Department of Surgery, Universidade do Vale do Sapucaí, Pouso Alegre, Brazil; ${ }^{3}$ Division of Plastic Surgery, Department of Surgery, Universidade Federal de São Paulo, São Paulo, Brazil; ${ }^{4}$ Department of Pathology, Universidade do Vale do Sapucaí, Pouso Alegre, Brazil; ${ }^{5}$ Department of Bioestatistics, Universidade do Vale do Sapucaí, Pouso Alegre, Brazil.

Email: *danifveiga@uol.com.br

Received July 24 $4^{\text {th }}$, 2012; revised August 26 ${ }^{\text {th }}, 2012$; accepted September $1^{\text {st }}, 2012$

\begin{abstract}
Background: The impact of breast appearance after breast cancer surgical treatment on patients' quality of life led to the development of the oncoplastic approach. However, studies reporting oncologic results associated with this treatment strategy are scarce. This cross-sectional study was designed to assess oncologic outcomes among patients who underwent oncoplastic surgery. Methods: A total of 190 breast cancer patients who underwent breast-conserving surgery were enrolled. Fifty of them underwent oncoplastic surgery and 140 had none breast reconstruction procedure (control group). All surgeries were performed by the same surgical team. Results: Groups were similar with regard to staging, histological type, grade of the tumor, presence of intraductal component, hormone receptors and nodal commitment. Patients in oncoplastic surgery group had larger tumors $(\rho=0.001)$ and more lymphovascular invasion $(\rho=$ 0.047). Further, a higher proportion of them underwent chemotherapy $(\rho=0.030)$. Follow-up time of control group was longer $(\rho=0.05)$, and these patients also had a longer relapse-free survival time $(\rho=0.001)$. Local recurrence rate was $5.8 \%(11 / 190)$ and it was significantly greater in the oncoplastic surgery group $(8 / 11, \rho=0.001)$. Time to local recurrence after surgery was longer in oncoplastic surgery group $(\rho=0.002)$. Overall, patients in oncoplastic surgery group were younger $(\rho=0.001)$, but at the time of local recurrence, patients in oncoplastic surgery group were older than those in control group $(\rho=0.0002)$. Conclusions: Among the studied patients, local recurrence rate was greater in those who underwent oncoplastic surgery.
\end{abstract}

Keywords: Breast Cancer; Mastectomy; Segmental; Breast Reconstruction; Local Neoplasm Recurrence

\section{Introduction}

Conservative surgery has become the standard surgical approach to early breast cancer since the studies of Veronesi et al. and Fisher et al. [1-3]. However, in the last two decades, conservative surgery has changed dramatically [4]. Changes include the conservative surgical approach to large tumors [5], the fact that resection of the skin and aponeurosis is no longer routinely performed, introduction of neoadjuvant chemotherapy, and immediate breast reconstruction, which has become part of the procedure.

Oncoplastic breast surgery, combining plastic surgery techniques and breast-conserving surgery, allows extensive resections and improves cosmetic results [6,7]. These procedures appear at first glance to be unnecessary,

*Corresponding author. since conservative surgery alone should provide satisfactory cosmetic results. However, in the past two decades, it has been observed that conservative surgery has produced poor cosmetic results in many cases, contradicting the present goal of breast cancer treatment, namely a better local control of the disease with presservation of the breast contours [5,8,9]. Furthermore, nowadays patients are increasingly cured of her disease, and less tolerant of poor aesthetic outcomes from breast surgery [10].

Local rotation flaps and other techniques provided the solution to breast reconstruction, resulting in better cosmetic results probably without interfering with oncologic outcomes. These procedures improve the quality of life and self-esteem of patients and usually do not require prolonged hospital stays $[5,11]$. However, it has been previously published that they could change parameters 
associated with disease behavior, the parenchymal architecture of the breast, and patterns of local recurrence, besides interfering with the clinical and mammographic follow-up of patients [12].

These issues have cast some doubts on the oncologic safety of this type of surgery [12]. As a consequence of the long natural history of breast cancer, and lack of long-term follow-up and comparative studies, the impact of oncoplastic breast surgery on local recurrence, metastasis, and global survival has not been fully investigated $[6,13,14]$. Evidence-based data are available only for studies comparing conservative surgery and mastectomy [15]. Literature on oncoplastic surgery and oncological outcomes is scarce and there is little data comparing oncoplastic surgery with standard breast conservative surgery from a single institution [10].

Therefore, the aim of this study was to assess the oncologic outcomes of breast cancer patients treated with conservative surgery.

\section{Patients and Methods}

This cross-sectional study was approved by the Research Ethics Committee of the Universidade do Vale do Sapucaí (UNIVÁS) and performed in accordance with the ethical standards of the 1964 Declaration of Helsinki and its succeeding revisions.

All breast cancer patients who underwent conservative surgery in the breast unit of Hospital das Clínicas Samuel Libânio between June 1997 and December 2008 were identified and were contacted to attend the breast ambulatory unity. Patients with a history of other malignant disease, not related to breast cancer, were not included in the sample. From 204 identified patients, we were not able to find 12, and two patients had thyroid tumors. Thus, 190 patients were enrolled. Except six patients, who had proven died due to breast cancer, all of them attended a medical consultation with one of the authors, and all signed a consent form.

All operations were performed by the same surgical team, consisting of two breast surgeons and one plastic surgeon. All pathological analyses were carried out by the pathologic anatomy service of the university hospital. Chemotherapy and hormone therapy were conducted at the clinical oncology service of the university hospital, following standard protocols. All patients received postoperative whole breast radiation therapy.

The patients were allocated into two groups: oncoplastic (OP) group, which included patients who underwent oncoplastic breast surgery $(\mathrm{n}=50)$, and control (C) group, which included patients who underwent conservative surgery without breast reconstruction $(n=140)$.

All patients underwent either conventional quadrantectomy or tumorectomy. Surgical specimens were mark- ed in three dimensions in the surgery room and handed over to the pathologist. The specimens were weighed and surgical margins were marked with India ink, so that the pathologist could determine whether tumor was present or absent at the margins. Specimens containing microcalcifications and non-palpable tumors were radiogramphed to confirm the presence of the lesion. Surgical margins of the tumors were evaluated by intraoperative frozen section analysis and, if necessary, resection was extended. Later, histopathological examination was performed on paraffin-embedded sections of all surgical margins. If a positive margin was detected, the patient underwent second-stage surgery and the margin was extended. Surgical margins free of tumor (greater than 2 $\mathrm{mm}$ ) were obtained in all cases [16].

Tumors were classified according to the pathologic tumor-node-metastasis TNM/pTNM classification of malignant tumors (Union for International Cancer Control, UICC 2002) [17]. The characteristics of the tumors are listed in Table 1.

All patients underwent axillary surgery. Axillary lymphadenectomy was routinely performed as a standard procedure until 2004, in all patients with invasive tumors who attended our institution. From then on, patent blue dye and gamma probe-guided sentinel lymph node biopsy has been carried out, and lymphadenectomy was performed only when the sentinel lymph node was either positive for metastases or not found or biopsy results were inconclusive (Table 1). Using axillary approach, a single incision was made for resection of tumors located in the upper outer quadrants of the breast, and separate incisions were made for tumors in other sites.

Oncoplastic surgery refers to several surgical techniques by which segments of breast tissue are removed to achieve wide margins around the tumor while the remaining glandular tissue is transposed to achieve the best possible aesthetic outcome [18-20]. Thus, in the OP group, local/regional flaps were performed; neither prostheses nor distant flaps were used. Eight patients underwent immediate contralateral symmetrization.

Mean hospital stay was 36 hours for both groups. Postoperative follow-up consisted of visits every 6 months to the same surgeons who performed the surgery. All patients underwent physical examination and routine mammograms at all follow-up visits. Other tests were performed only in the case of clinical indication.

\section{Statistical Analysis}

Non-parametric tests were used for the statistical analysis. The Mann-Whitney U test was used to compare age, follow-up time, relapse-free survival time, tumor size, specimen weight and number of committed lymph nodes between groups. Chi-square test or Fisher test were used 
Table 1. Oncologic data of patients who underwent oncoplastic surgery (OP group) or conservative surgery without breast reconstruction (C group) and statistical results.

\begin{tabular}{|c|c|c|c|}
\hline \multirow{2}{*}{ TNM/pTNM staging system [16] } & OP group $(n=50)$ & $C$ group $(n=140)$ & \multirow{2}{*}{ OP $\times$ C Chi-square test or Fisher test } \\
\hline & n (\%) & n (\%) & \\
\hline Stage 0 & $2(4 \%)$ & 13 (9\%) & \multirow{5}{*}{$\chi^{2}=7.97 ; \rho=0.093$} \\
\hline Stage I & $16(32 \%)$ & $56(40 \%)$ & \\
\hline Stage II & $22(44 \%)$ & $42(30 \%)$ & \\
\hline Stage III & $10(20 \%)$ & $26(19 \%)$ & \\
\hline TxN0M0 & 0 & $3(2 \%)$ & \\
\hline \multicolumn{4}{|l|}{ Histological type } \\
\hline Ductal carcinoma in situ & $2(4 \%)$ & $13(9 \%)$ & \multirow{3}{*}{$\chi^{2}=2.33 ; \rho=0.304$} \\
\hline Invasive ductal carcinoma & 47 (94\%) & $120(86 \%)$ & \\
\hline Lobular invasive carcinoma & $1(2 \%)$ & $7(5 \%)$ & \\
\hline \multicolumn{4}{|l|}{ Grade } \\
\hline Grade I & $12(24 \%)$ & $22(16 \%)$ & \multirow{3}{*}{$\chi^{2}=2.92 ; \rho=0.233$} \\
\hline Grade II & $22(44 \%)$ & $56(40 \%)$ & \\
\hline Grade III & $16(32 \%)$ & $62(44 \%)$ & \\
\hline \multicolumn{4}{|l|}{ Axillary approach } \\
\hline Negative SLNB ${ }^{a}$ & $17(34 \%)$ & $50(36 \%)$ & $\chi^{2}=0.05 ; \rho=0.964$ \\
\hline Axillary dissection & $33(66 \%)$ & $90(64 \%)$ & \\
\hline Chemotherapy & $42(84 \%)$ & $93(66 \%)$ & $\chi^{2}=5.53 ; \rho=0.030^{\mathrm{b}}$ \\
\hline Hormone therapy & 47 (94\%) & 125 (89\%) & $\rho=0.410$ \\
\hline
\end{tabular}

${ }^{\mathrm{a}}$ Sentinel lymph node biopsy; ${ }^{\mathrm{b}}$ Statistical significance.

to compare oncologic data, occurrence of metastases, recurrence, and death rate between groups. Log-rank test was used to compare groups with regard to time to events (local recurrence, contralateral breast tumor, distant metastases, death). This test was also used to compare age at local recurrence between groups.

Statistical analysis was carried out using the Statistical Package for the Social Sciences (SPSS) version 18.0 for Windows (SPSS Inc., Chicago, IL). All statistical tests were performed at a significance level of $0.05(\rho<0.05)$.

\section{Results}

The median age of patients was 48.5 years (range, 33 75 years) in the OP group, and 53.0 years (range, 23 - 83 years) in the $\mathrm{C}$ group, with significant difference between groups $(\rho=0.001)$. Oncologic data for the patients are listed in Table 1.

The median follow-up time was 38.4 months (range, 6 - 117 months) in the OP group, and 53.3 months (range, 6 - 148 months) in the $\mathrm{C}$ group, significantly longer to CS group $(\rho=0.05)$. The median relapse-free survival time was 26 months (range, 10 - 105 months) in the OP group, and 46 months (range, 6 - 136 months) in the $\mathrm{C}$ group $(\rho=0.001)$.

The most common localization was the outer quadrants (80\% and 66\% in OP and C groups respectively). Tumors ranged in size from 0.7 to $6.0 \mathrm{~cm}$ (median 2.2 $\mathrm{cm}$ ) in OP group and from 0.1 to $7.5 \mathrm{~cm}$ (median $1.6 \mathrm{~cm}$ ) in $\mathrm{C}$ group $(\rho=0.001)$. The median specimen weight was $80 \mathrm{~g}$ (range, 5 - $495 \mathrm{~g}$ ) in the OP group, and $45 \mathrm{~g}$ (range, $6-233 \mathrm{~g}$ in the $\mathrm{C}$ group $(\rho=0.000)$. The number of committed lymph nodes ranged from 0 to 24 (median 1 lymph node) in OP group and from 0 to 32 (median 0 ) in C group $(\rho=0.150)$.

Some tumor characteristics were not available for all the 190 patients, but we used the available data to compare both groups. Groups did not differ with regard to the presence of intraductal component $(\rho=0.088)$, estrogen receptors $(\rho=1.000)$, progesterone receptors $(\rho=1.000)$, HER 2 status $(\rho=1.000)$, Ki $67(\rho=0.061)$ and TP $53(\rho$ $=0.151)$, but OP group had more lymphovascular inva$\operatorname{sion}(\rho=0.047)$. 
Eleven patients (5.8\%) had local recurrence, eight of whom were in the OP group $(\rho=0.001)$. Except for one patient in the OP group whose tumor relapsed to high grade carcinoma in situ and one patient in the $\mathrm{C}$ group who developed Paget's disease (carcinoma in situ), all recurrences were diagnosed as invasive carcinoma. Groups did not differ with regard to other events, such as contralateral breast tumor, distant metastases or death due to breast cancer (Table 2).

Of the eight patients with recurrence in the OP group, two had undergone reduction mammaplasty as oncoplastic surgery, and six local flaps. Three of these patients had surgical margins extended during first-stage surgery.

Eight patients (all in the $\mathrm{C}$ group) with positive margins on histopathological examination of paraffin-embedded sections underwent second-stage surgery to complete tumor clearance. All eight patients had no recurrence.

The median time to local recurrence after surgery was 36 months (range, 12 - 54 months) in the OP group and 24 months (range, 15 - 127 months) in the $\mathrm{C}$ group ( $\rho=$ 0.0002). There were no significant differences between groups regarding time to contralateral breast recurrence, distant metastases or death rate (Table 3).

Patients' age at the time of local recurrence ranged from 33 to 56 years (median 46.5 years) in the in the OP group and from 23 to 57 years (median 36.0 years) in the C group $(\rho=0.0002)$.

Of the 11 patients who developed ipsilateral breast tumor recurrence, only one patient in the OP group died.
Three other patients (two in the OP group and one in the C group), who developed meningeal metastasis, bone and lung metastases, and disseminated metastasis, respecttively, had a poor short-term prognosis.

All patients had mammography every six months during the follow-up period. However, in most cases, local tumor recurrence was detected on physical examination as a nodule or mass in the operated breast.

\section{Discussion}

Although cosmetic results following conservative surgery for breast cancer are considered important [8,9], one must keep in mind that the primary goal of the treatment is the oncologic outcome.

In the present study, the local recurrence rate was $5.8 \%$ during the follow-up period (range, $12-127$ months). It is difficult to compare our findings with those of previous studies because of different sample populations and also because classical studies have excluded large tumors. The results of the National Surgical Adjuvant Breast and Bowel Project (NSABP-protocol B-04) showed a cumulative local recurrence rate of $14.3 \%$, excluding tumors greater than $4 \mathrm{~cm}$ [3]. The Curie Institute reported a $9.4 \%$ recurrence rate after a five-year follow-up [5], and the Milan I study, which excluded tumors greater than $2 \mathrm{~cm}$, reported a local recurrence rate of $8.8 \%$ after a 20 -year follow-up [2]. More recently, Rietjens et al. reported a recurrence rate of 3\% in Stage pT2 or lesser tumors. These authors did not report local recurrence for tumors less than $2 \mathrm{~cm}$ [6]. Ueda et al.

Table 2. Follow-up data and comparison of oncologic outcomes of patients in the oncoplastic (OP) and control (C) groups.

\begin{tabular}{lccc}
\hline Oncologic outcomes & OP group (n = 50) & C group (n= 140) & OP vs. C \\
\hline & $\mathrm{n}(\%)$ & $\mathrm{n}(\%)$ & Fisher test \\
Local recurrence & $8(16 \%)$ & $3(2 \%)$ & $\rho=0.001^{\mathrm{a}}$ \\
Contralateral breast recurrence & $2(4 \%)$ & $3(2 \%)$ & $\rho=0.607$ \\
Distant metastases & $4(8 \%)$ & $7(5 \%)$ & $\rho=0.484$ \\
Death from breast cancer & $2(4 \%)$ & $4(3 \%)$ & $\rho=0.649$ \\
\hline
\end{tabular}

${ }^{\mathrm{a}}$ Statistical significance.

Table 3. Comparison of patients in the oncoplastic (OP) and control (C) groups with regard to time to breast cancer events.

\begin{tabular}{lccc}
\hline & \multicolumn{1}{c}{ Time to event (months) } & Log-rank test \\
\hline Event & OP group Median (range) & C group Median (range) & $24(15-127)$ \\
Local recurrence & $36(12-54)$ & $57(55-62)$ & $0.0002^{\mathrm{a}}$ \\
Contralateral breast tumor & $21(14-27)$ & $36(21-64)$ & 0.4671 \\
Distant metastases & $21(12-26)$ & $31(22-62)$ \\
\hline
\end{tabular}

${ }^{\mathrm{a}}$ Statistical significance. 
reported a $2 \%$ rate of local recurrence in breast-conserving surgery with no breast reconstruction [4], and a recent meta-analysis presented a recurrence (locoregional or distant) rate of $0.52 \%$ per year, in a 10 -years follow-up [21]. Fitoussi et al. found a $6.8 \%$ rate of local recurrence after oncoplastic surgery, including T3 tumors [22].

In the present study, the follow-up period was signifycantly longer in the $\mathrm{C}$ group $(\rho=0.05)$, but local recurrence rate was higher in the OP group $(\rho<0.001)$. This was an unexpected finding, because most previous studies have reported similar oncologic outcomes for patients who underwent conservative surgery and those who underwent oncoplastic surgery [6]. However, Losken et al. in a study that evaluated long-term postoperative cancer surveillance in patients who underwent either conservative surgery or oncoplastic surgery, reported that a larger number of biopsies were performed during the follow-up in the oncoplastic surgery group (one biopsy every 4 years) compared with the conservative surgery group (one biopsy every 33 years) [12].

A possible explanation for the greater number of recurrences in the OP group may be the age of the patients (48.5 years vs 53 years, $\rho<0.001$ ), since age is an important prognostic factor. However, when we compared both groups with regard to age at local recurrence, we find older patients in OP group $(\rho=0.0002)$. Other authors have also reported the lower mean age of oncoplastic surgery patients, but have not discussed the influence of age on treatment outcome [12,13,23].

Both groups were similar with regard to staging, histological type, grade of the tumor, presence of intraductal component, hormone receptors and nodal commitment. There was no significant association of tumor staging and histological grade with recurrences. In the OP group, recurrences were observed in three Stage I patients, three Stage IIA, one Stage IIIA, and one Stage IIIC patient. In the CS group, recurrences were found in two Stage I patients and one Stage IIA patient. Recurrences were most commonly found in Stage pT1 patients (36.4\%, 4/11), contrary to the findings of Rietjens et al. who did not report local recurrences in Stage pT1 patients [6].

In the present study, patients with all sizes of tumors (including 36 tumors $5 \mathrm{~cm}$ or larger) underwent conservative surgery, and patients in OP group had larger tumors $(\rho=0.001)$. However, similar to findings of Chakravorty et al., it was observed that recurrences were not associated with the large tumors [10]. This is in agreement with the findings obtained by Khanna et al. when evaluating 68 patients with tumors ranging in size from 4 to $12 \mathrm{~cm}$ who underwent conservative surgery for breast cancer. These authors concluded that conservative surgery may be used in patients with tumors $4 \mathrm{~cm}$ or larger without compromise in locoregional control or survival
[24]. Clough et al. conducted a study in patients with tumors larger than $5 \mathrm{~cm}$ who were treated conservatively and found no negative results associated with tumor size [5]. In a study on tumor beds in patients who underwent conservative treatment, Veronesi et al. concluded that residual disease was the cause of recurrence and that residual disease was not associated with age group or tumor size [23].

Patients in OP group had larger tumors $(\rho=0.001)$ and more lymphovascular invasion $(\rho=0.047)$. Padera et al. in a study on liver tumors in mice, reported that functional lymphatics in the tumor margin are sufficient for lymphatic metastasis, regardless of the size of the tumor [25]. Unfortunately, we were not able to study the association between lymphovascular invasion and local recurrence, because of the high number of missing data for this variable, and we must recognize that this is a major flaw in this study. Removing the primary tumor with negative surgical margins is the most important determinant of local recurrence [23,26]. In our study, margins free of tumor were obtained in all cases. Eight patients with positive margins on histopathological examination of paraffin-embedded sections underwent second-stage surgery to complete tumor clearance. None of these patients had local recurrences. All the patients who required second-stage surgery were in CS group. This fact, associated to significant larger tissue resections in OP group ( $\rho=0.000)$, may suggest that the surgical margins in the OP group were wider. However, it had no positive impact on the results. Three of the eight patients with local recurrence in the OP group had surgical margins extended during first-stage surgery. In one of the cases, the extended posterior margin (aponeurosis of the major pectoralis) revealed a neoplastic growth in the lumen of a lymphatic vessel.

Veronesi et al. found no differences in mortality when comparing conservative surgery and mastectomy, despite the fact that the number of local recurrences were greater in patients who underwent conservative surgery [1,2]. In another study, Veronesi et al. reported that younger patients with local recurrence, especially those with early ( $<2$ years) local failure, were at increased risk for distant metastases [23]. In our study, most patients who developed recurrence had invasive recurrence (9 of 11 patients), which is associated with a poor prognosis [23]. The majority of the cases of local recurrence were detected on physical examination. This highlights the importance of patient education and follow-up examinations conducted by an experienced physician.

In the present study, all patients underwent radiotherapy. It has been demonstrated that radiotherapy after conservative breast surgery significantly reduces the rate of local recurrence $[27,28]$. The Early Breast Cancer Trialists’ Collaborative Group (EBCTCG) performed a meta- 
analysis of data from more than 10,000 patients of radiotherapy versus no radiotherapy after breast-conserving surgery. In addition to the significant absolute risk reduction of the 10-year risk of recurrence in patients who underwent radiotherapy, it was observed that radiotherapy also reduced breast cancer death, with a 15-year absolute risk reduction of 3.8\% (likely one breast cancer death avoided for every four recurrences avoided by radiotherapy) [21].

Our findings must be interpreted in the context of several limitations. A major one is the lack of randomization, which can have led to a selection bias. Indeed, patients in OP group were younger, had larger tumors, had more lymphovascular invasion, and a higher proportion of them underwent chemotherapy. Despite patients' age be a potential source of selection bias, groups did not differ with regard to preoperative tumor staging [17]. Further, several tumors characteristics are unknown at the moment of patient selection, such as the accurate tumor size, presence of intraductal component and lymphovascular invasion, among others.

Another limitation of this study is the sample size. Despite we contacted all the patients who underwent conservative surgery in our hospital in 11 years, the total number of operations was relatively low. A larger sample, perhaps obtained from a multicenter study, would enhance the power of this study and better supports its findings. On the other hand, positive aspects of this study include the fact that all patients were operated and followed-up over a relatively long period of time (global mean, 46.9 months) by the same surgeons.

\section{Conclusion}

In the present study, local recurrence rate was greater in patients who received oncoplastic surgery. Our results regarding the oncologic safety of the oncoplastic surgery do not agree with the literature, however, our study design does not allow any definitive conclusion. While not conclusive, this study could be a starting point to clarify important issues. Despite technical and ethical hurdles to randomize breast cancer patients (especially young patients) to determine whether they would receive oncoplastic surgery, prospective randomized clinical trials should be conducted to definitively establish (or not) the safety of oncoplastic surgery for breast cancer.

\section{REFERENCES}

[1] U. Veronesi, A. Banfi, M. Del Vecchio, et al., "Comparison of Halsted Mastectomy with Quadrantectomy, Axillary Dissection, and Radiotherapy in Early Breast Cancer: Long-Term Results," European Journal of Cancer and Clinical Oncology, Vol. 22, No. 9, 1986, pp. 1085-1089. doi:10.1016/0277-5379(86)90011-8
[2] U. Veronesi, N. Cascinelli, L. Mariani, et al., "TwentyYear Follow-Up of a Randomized Study Comparing Breast Conserving Surgery with Radical Mastectomy for Early Breast Cancer," The New England Journal of Medicine, Vol. 347, No. 16, 2002, pp. 1227-1232. doi:10.1056/NEJMoa020989

[3] B. Fisher, S. Anderson, J. Bryant, et al., "Twenty-Year Follow-Up of a Randomized Trial Comparing Total Mastectomy, Lumpectomy and Lumpectomy plus Irradiation for the Treatment of Invasive Breast Cancer," The New England Journal of Medicine, Vol. 347, No. 16, 2002, pp. 1233-1241. doi:10.1056/NEJMoa022152

[4] S. Ueda, Y. Tamaki, K. Yano, et al., "Cosmetic Outcome and Patient Satisfaction after Skin-Sparing Mastectomy for Breast Cancer with Immediate Reconstruction of the Breast,” Surgery, Vol. 143, No. 3, 2008, pp. 414-425. doi:10.1016/j.surg.2007.10.006

[5] K. B. Clough, J. S. Lewis, B. Couturaud, A. Fitoussi, C. Nos and M. C. Falcou, "Oncoplastic Techniques Allow Extensive Resections for Breast Conserving Therapy of Breast Carcinomas,” Annals of Surgery, Vol. 237, No. 2003, pp. 26-34. doi:10.1097/00000658-200301000-00005

[6] M. Rietjens, C. A. Urban, P. C. Rey, et al., "Long-Term Oncological Results of Breast Conservative Treatment with Oncoplastic Surgery,” Breast, Vol. 16, No. 4, 2007, pp. 387-395. doi:10.1016/j.breast.2007.01.008

[7] B. O. Anderson, R. Masetti and M. J. Silverstein, “Oncoplastic Approaches to Partial Mastectomy: An Overview of Volume-Displacement Techniques,” The Lancet Oncology, Vol. 6, No. 3, 2005, pp. 145-147. doi:10.1016/S1470-2045(05)01765-1

[8] D. F. Veiga, J. Veiga-Filho, L. M. Ribeiro, et al., "Evaluations of Aesthetic Outcomes of Oncoplastic Surgery By Surgeons of Different Gender and Specialty: A Prospective Controlled Study,” Breast, Vol. 20, No. 5, 2011, pp. 407-412. doi:10.1016/j.breast.2011.04.001

[9] A. K. Bajaj, P. S. Kon, K. C. Oberg and D. A. G. Miles, "Aesthetic Outcomes in Patients Undergoing Breast Conservation Therapy for the Treatment of Localized Breast Cancer," Plastic and Reconstructive Surgery, Vol. 114, No. 6, 2004, pp. 1442-1449. doi:10.1097/01.PRS.0000138813.64478.A7

[10] A. Chakravorty, A. K. Shrestha, N. Sanmugalingam, et al., "How Safe is Oncoplastic Breast Conservation? Comparative Analysis with Standard Breast Conserving Surgery," European Journal of Surgical Oncology, Vol. 38, No. 5, 2012, pp. 395-398.doi:10.1016/j.ejso.2012.02.186

[11] D. F. Veiga, J. Veiga-Filho, L. M. Ribeiro, et al., "Quality of Life and Self-Esteem Outcomes after Oncoplastic Breast-Conserving Surgery,” Plastic and Reconstructive Surgery, Vol. 125, No. 3, 2010, pp. 811-817. doi:10.1097/PRS.0b013e3181ccdac5

[12] A. Losken, T. G. Schaefer, M. Newell and T. M. Styblo, "The Impact of Partial Breast Reconstruction Using Reduction Techniques on Postoperative Cancer Surveillance," Plastic and Reconstructive Surgery, Vol. 124, No. 1, 2009, pp. 9-17. doi:10.1097/PRS.0b013e3181ab10e5

[13] P. L. Giacalone, P. Roger, O. Dubon, et al., "Comparative 
Study of the Accuracy of Breast Resection in Oncoplastic Surgery and Quadrantectomy in Breast Cancer," Annals of Surgical Oncology, Vol. 14, No. 2, 2007, pp. 605-614. doi:10.1245/s10434-006-9098-5

[14] D. H. Roukos, A. M. Kappas and N. J. Agnantis, "Perspectives and Risks of Breast-Conservation Therapy for Breast Cancer," Annals of Surgical Oncology, Vol. 10, No. 7, 2003, pp. 718-721. doi:10.1245/ASO.2003.05.925

[15] J. Hoffmann and D. Wallwiener, “Classifying Breast Cancer Surgery: A Novel Complexity-Based System for Oncological, Oncoplastic and Reconstructive Procedures, and Proof of Principle by Analysis of 1225 Operations in 1166 Patients,” BMC Cancer, Vol. 9, 2009, p. 108. doi:10.1186/1471-2407-9-108

[16] B. Fowble, “The Significance of Resection Margin Status in Patients with Early-Stage Invasive Cancer Treated with Breast-Conservation Therapy," The Breast Journal, Vol. 4, 1998, pp. 126-131. doi:10.1046/j.1524-4741.1998.420126.x

[17] S. E. Singletary, C. Allred, P. Ashley, et al., "Revision of the American Joint Committee on Cancer Staging System for Breast Cancer," Journal of Clinical Oncology, Vol. 20, No. 17, 2002, pp. 3628-3636. doi:10.1200/JCO.2002.02.026

[18] J. Kolias, G. Davies, M. A. Bochner and P. G. Gill, "Clinical Impact of Oncoplastic Surgery in a Specialist Breast Practice," ANZ Journal of Surgery, Vol. 78, No. 4, 2008, pp. 269-272. doi:10.1111/j.1445-2197.2008.04435.x

[19] B. O. Anderson, R. Masetti and M. J. Silverstein, “Oncoplastic Approaches to Partial Mastectomy: An Overview of Volume-Displacement Techniques," The Lancet Oncology, Vol. 6, No. 3, 2005, pp. 145-147. doi:10.1016/S1470-2045(05)01765-1

[20] K. S. Asgeirsson, T. Rasheed, S. J. McCulley and R. D. Macmillan, "Oncological and Cosmetic Outcomes of Oncoplastic Breast Conserving Surgery,” European Journal of Surgical Oncology, Vol. 31, No. 8, 2005, pp. 817823. doi:10.1016/j.ejso.2005.05.010

[21] Early Breast Cancer Trialists' Collaborative Group, S.
Darby, P. McGale, et al., "Effect of Radiotherapy after Breast-Conserving Surgery on 10-Year Recurrence and 15-Year Breast Cancer Death: Meta-Analysis of Individual Patient Data for 10,801 Women in 17 Randomised Trials,” Lancet, Vol. 378, No. 9804, 2011, pp. 1707-1716. doi:10.1016/S0140-6736(11)61629-2

[22] A. D. Fitoussi, M. G. Berry, F. Famà, et al., "Oncoplastic Breast Surgery for Cancer: Analysis of 540 Consecutive Cases,” Plastic and Reconstructive Surgery, Vol. 125, No. 2, 2010, pp. 454-462. doi:10.1097/PRS.0b013e3181c82d3e

[23] U. Veronesi, E. Marubini, M. Del Vecchio, et al., "Local Recurrences and Distant Metastases after Conservative Breast Cancer Treatments: Partly Independent Events,” Journal of the National Cancer Institute, Vol. 87, No. 1, 1995, pp. 19-27. doi:10.1093/jnci/87.1.19

[24] M. M. Khanna, R. J. Mark, M. J. Silverstein, G. Juillard, B. Lewinsky and A. E. Giuliano, "Breast Conservation Management of Breast Tumors $4 \mathrm{~cm}$ or Larger," Archives of Surgery, Vol. 127, No. 9, 1992, pp. 1038-1041. doi:10.1001/archsurg.1992.01420090042007

[25] T. P. Padera, A. Kadambi, E. di Tomaso, et al., "Lymphatic Metastasis in the Absence of Functional Intratumor Lymphatics," Science, Vol. 296, No. 5574, 2002, pp. 1883-1886. doi:10.1126/science. 1071420

[26] C. Atalay and C. Irkkan, "Predictive Factors for Residual Disease in Re-Excision Specimens after Breast-Conserving Surgery," The Breast Journal, Vol. 18, No. 4, 2012, pp. 339-344. doi:10.1111/j.1524-4741.2012.01249.x

[27] M. Amichetti and C. Vidali, "Radiotherapy after Conservative Surgery in Ductal Carcinoma in Situ of the Breast: A Review," International Journal of Surgical Oncology, Vol. 2012, 2012. doi:10.1155/2012/635404

[28] G. G. Kimmick, F. Camacho, W. Hwang, H. Mackley, J. Stewart and R. T. Anderson, "Adjuvant Radiation and Outcomes after Breast Conserving Surgery in Publicly Insured Patients,” Journal of Geriatric Oncology, Vol. 3, No. 2, 2012, pp. 138-146. doi:10.1016/j.jgo.2012.01.004 